\title{
Apigenin suppresses the stem cell-like properties of triple-negative breast cancer cells by inhibiting YAP/TAZ activity
}

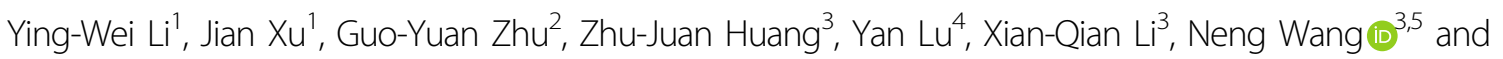
Feng-Xue Zhang ${ }^{3}$

\begin{abstract}
Triple-negative breast cancer (TNBC) remains a clinical challenge because of the absence of effective therapeutic targets. In TNBC, overexpression of YAP and TAZ correlates with bioactivities of cancer stem cells (CSCs), high histological grade, resistance to chemotherapy, and metastasis. Thus, YAP/TAZ may serve as potential therapeutic targets in TNBC. To identify YAP/TAZ inhibitors, in previous experiments, we screened a library of natural compounds by using YAP/TAZ luciferase reporter assay and identified apigenin as a potential inhibitor. In this study, we demonstrated that apigenin significantly suppressed the proliferation and migration of TNBC cells. Furthermore, we demonstrated that apigenin inhibited stemness features of TNBC cells in both in vitro and in vivo assays. Our mechanism study demonstrated that apigenin decreased YAP/TAZ activity and the expression of target genes, such as CTGF and CYR61, in TNBC cells. We also showed that apigenin disrupted the YAP/TAZ-TEADs protein-protein interaction and decreased expression of TAZ sensitized TNBC cells to apigenin treatment. Collectively, our studies suggest that apigenin is a promising therapeutic agent for the treatment of TNBC patients with high YAP/TAZ activity.
\end{abstract}

\section{Introduction}

Breast cancer is the most frequently diagnosed cancer in women worldwide ${ }^{1,2}$. It is highly heterogeneous and clinically classified into different subtypes based on the status of receptors ${ }^{3}$. Triple-negative breast cancer (TNBC) is one of the most aggressive breast cancer subtypes and constitutes around $15-20 \%$ of breast cancer cases ${ }^{4}$. Because of the lack of therapeutic targets, patients diagnosed with this type of breast cancer have a poor prognosis, along with a high rate of recurrence after

\footnotetext{
Correspondence: Ying-Wei Li (yingweilyw@yahoo.com)

Feng-Xue Zhang (zhangfengxue@gzucm.edu.cn)

${ }^{1}$ Tropical Medicine Institute, Guangzhou University of Chinese Medicine, 510006 Guangzhou, P.R. China

${ }^{2}$ State Key Laboratory of Quality Research in Chinese Medicine, Macau Institute for Applied Research in Medicine and Health, Macau University of Science and Technology, Macau, P.R. China

Full list of author information is available at the end of the article. Edited by I. Amelio
}

chemotherapy ${ }^{5,6}$. Hence, the development of effective treatments for TNBC is an important unmet medical need.

YAP and TAZ, two main downstream effectors of the Hippo pathway, are overexpressed or aberrantly activated in various malignancies ${ }^{7,8}$. Abnormal activation of YAP/ TAZ is involved in several biological processes, such as epithelial-mesenchymal transition (EMT), tumor metastasis, and tumorigenesis ${ }^{9-13}$. The most recent studies also demonstrate that YAP/TAZ is required to sustain the selfrenewal and tumor-initiation capacities of cancer stem cells $(\mathrm{CSCs})^{14,15}$. Particularly in TNBC, high YAP/TAZ activity correlates with bioactivities of CSCs, high-grade histology, and metastasis ${ }^{16-21}$. Thus, identifying new potential therapeutic agents that inhibit YAP/TAZ activity is meaningful for the treatment of TNBC.

Natural products and their derivatives have been used as anticancer agents and present a potential source of new drugs to combat cancer ${ }^{22-24}$. To identify potential YAP/ TAZ inhibitors, we previously performed a YAP/TAZ

\section{(c) 2018 The Author(s)}

(c) (i) Open Access This article is licensed under a Creative Commons Attribution 4.0 International License, which permits use, sharing, adaptation, distribution and reproduction in any medium or format, as long as you give appropriate credit to the original author(s) and the source, provide a link to the Creative Commons license, and indicate if changes were made. The images or other third party material in this article are included in the article's Creative Commons license, unless indicated otherwise in a credit line to the material. If material is not included in the article's Creative Commons license and your intended use is not permitted by statutory regulation or exceeds the permitted use, you will need to obtain permission directly from the copyright holder. To view a copy of this license, visit http://creativecommons.org/licenses/by/4.0/. 
luciferase reporter activity-based screening of a library of natural products (Selleckchem, Houston, TX, USA), and found that apigenin decreased YAP/TAZ activity. In this study, we demonstrated that apigenin significantly suppressed the migration and CSCs properties of TNBC cells. We found that apigenin acted at least partially by inhibiting the YAP/TAZ-TEADs complex activity. These results confirmed that apigenin was a promising agent for the treatment of TNBC patients with high YAP/TAZ activity.

\section{Results}

\section{Apigenin inhibited cell viability in TNBC cells}

We used the SRB protein assay to investigate the effect of apigenin on the proliferation of TNBC cells and found that apigenin inhibited cell proliferation in a dose- and time-dependent manner (Fig. 1b, c). The IC50 values of apigenin in MDA-MB-231 and MDA-MB-436 cells were approximately 33 and $30 \mu \mathrm{M}$, respectively, at $72 \mathrm{~h}$. As shown in Supplementary Fig. 1, apigenin was relatively nontoxic to normal human breast cells. We also examined whether apigenin might affect the ability of TNBC cells to form colonies and found that apigenin indeed significantly reduced the rate of colony formation in both TNBC cell lines (Fig. 1d, e).

\section{Apigenin attenuated migration in TNBC cells}

We used wound-healing assays to evaluate the effects of apigenin on the migration of TNBC cells. Our results demonstrated that apigenin reduced gap closure at concentrations of 10 and $20 \mu \mathrm{M}$ (Fig. 2a, b). We also conducted transwell migration assays on TNBC cells to confirm the above finding. As shown in Fig. 2c, apigenin treatment decreased the number of migrated cells in a dose-dependent manner. Statistical analysis indicated that apigenin caused a significant decrease in the migration of MDA-MB-231 and MDA-MB-436 cells (Fig. 2d).

\section{Apigenin suppressed the stem cell-like properties and tumorigenic potential of TNBC cells}

We examined the anti-CSCs effects of apigenin over a range of concentrations that did not cause massive cell death $(0-20 \mu \mathrm{M})$. We first investigated whether apigenin could decrease the percentage of the $\mathrm{CD} 44^{+} / \mathrm{CD} 24^{-}$subpopulation in TNBC cells. As shown in Fig. 3a, b, apigenin treatment decreased the $\mathrm{CD} 44^{+} / \mathrm{CD} 24^{-} \mathrm{CSC}$ subpopulations in

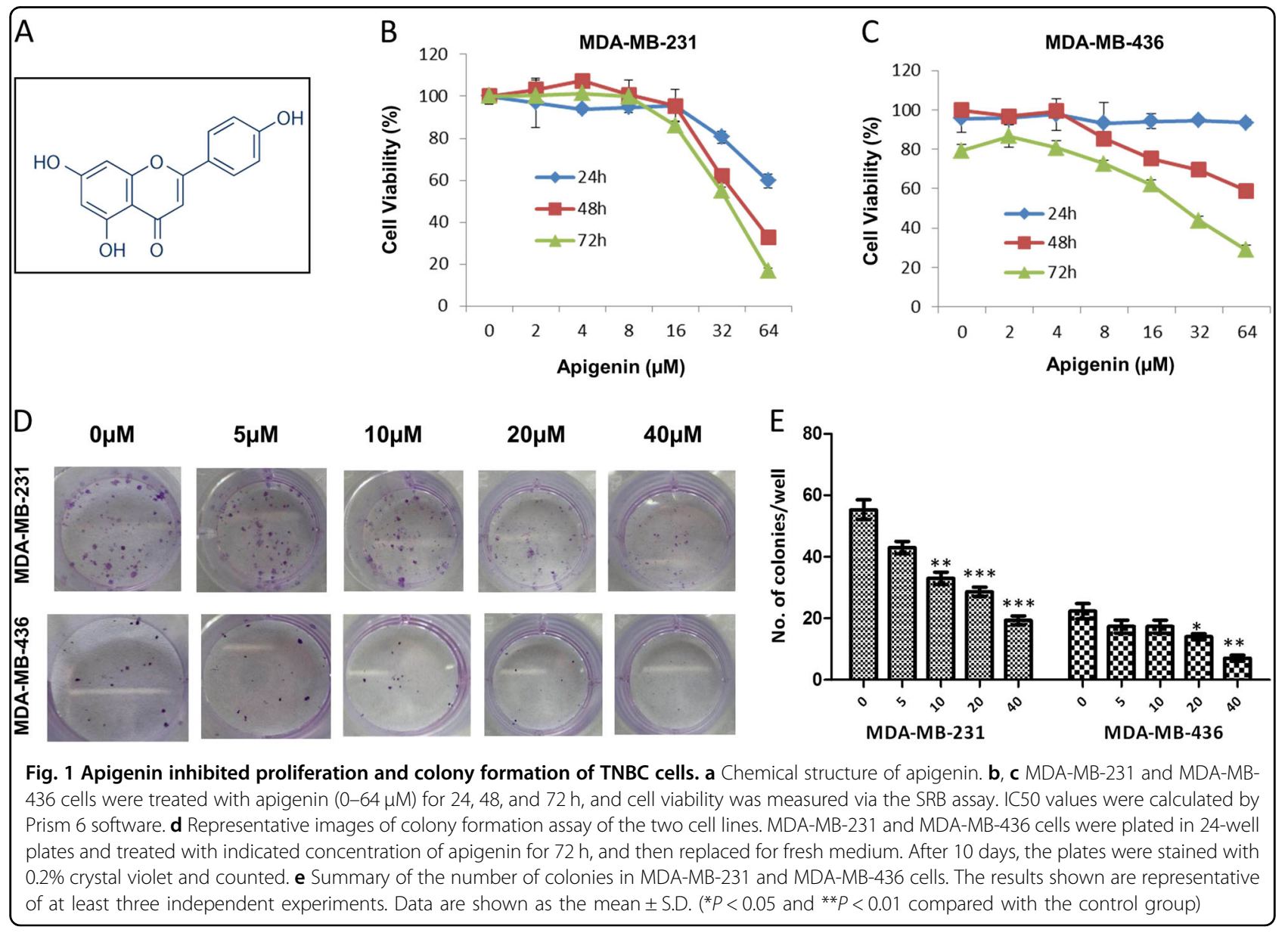



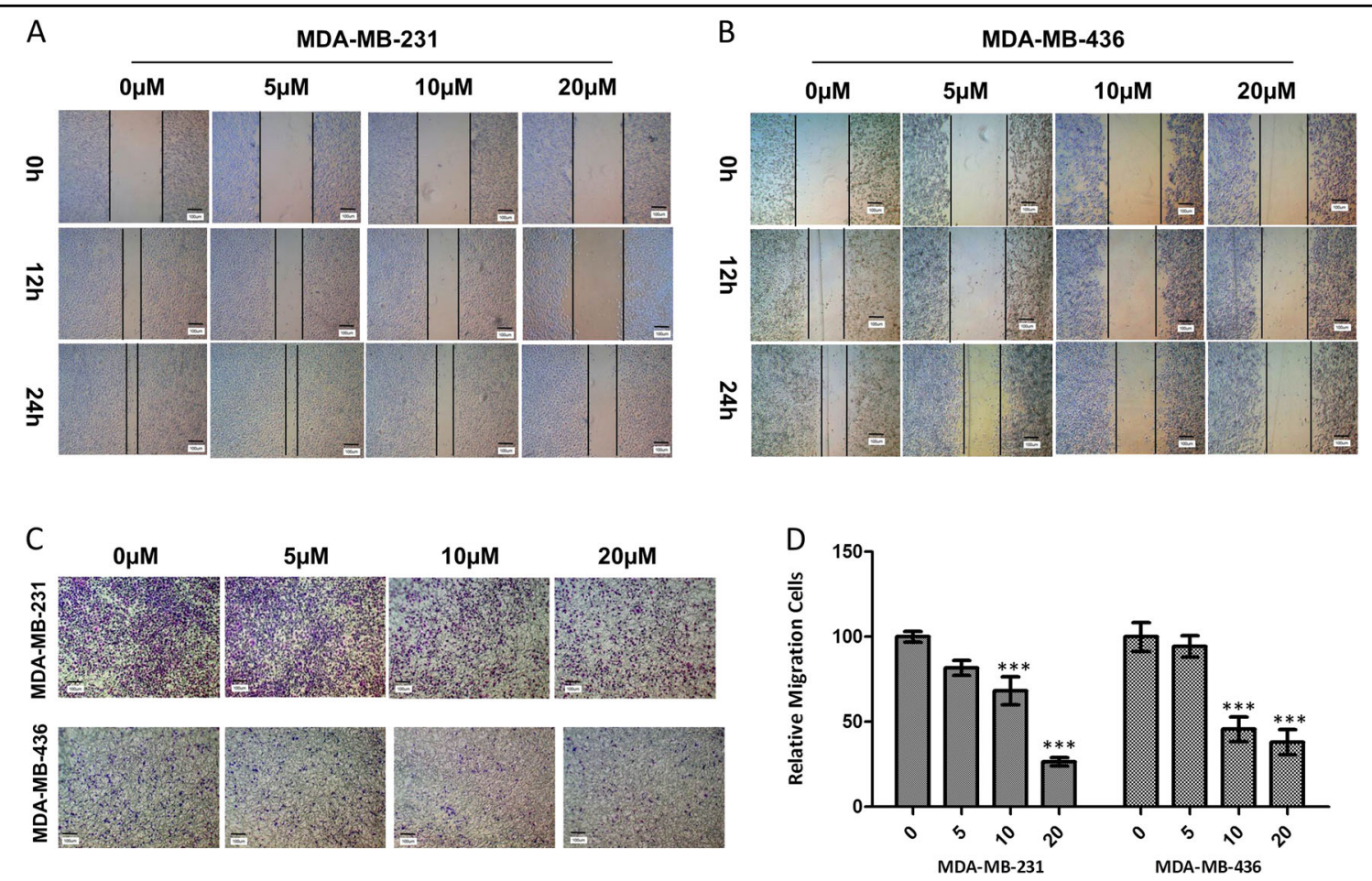

Fig. 2 Apigenin attenuated migration of TNBC cells. $\mathbf{a}$, $\mathbf{b}$ Representative images from the wound-healing assay of MDA-MB-231 and MDA-MB-436 cells are displayed for 12 and $24 \mathrm{~h}$. c Representative images of the transwell migration assay are shown for $24 \mathrm{~h}$ after cells seeding. $\mathbf{d}$ Statistical analysis of relative migrating cell numbers. The results shown are representative of at least three independent experiments. Data are shown as the means \pm S.D. ${ }^{* *} P<0.01$ and ${ }^{* * *} P<0.001$ compared with the control group)

MDA-MB-231 and MDA-MB-436 cells. Consistent with these findings, we observed that apigenin significantly decreased the number of mammospheres formed from both cell lines, indicating a reduction in self-renewal capability (Fig. 3c, d). These results indicated that apigenin decreased CSC-like traits on TNBC cells in vitro.

We also examined the effects of apigenin on the tumorinitiating properties of TNBC cells using an in vivo limited dilution assay. As shown in Fig. $4 \mathrm{a}, 1 \times 10^{6}$ and $1 \times 10^{5}$ MDA-MB-231 cells formed tumor xenografts with $100 \%$ efficiency in the control group, but the tumor formation efficiency of the apigenin-treated group decreased to 50\% and $20 \%$, respectively. When cells were seeded at a density of $1 \times 10^{4}$ cells per site, the tumor formation efficiency in the apigenin-treated group decreased to $0 \%$, whereas the control group retained $75 \%$ of its efficiency. Additionally, the onset of tumor growth in the apigenin-treated group was delayed compared with the control, DMSO-treated group (Fig. 4b). Apigenin treatment also significantly decreased tumor volumes and weights (Fig. 4c-e).

Apigenin reversed the malignant phenotype of TNBC cells by inhibiting YAP/TAZ transcription activities

To explore whether apigenin inhibited YAP/TAZ transcriptional activity in TNBC cells, we used the luciferase reporter assay. Our results demonstrated that apigenin significantly decreased YAP/TAZ activity in TNBC cells (Fig. 5a). To further confirm that apigenin inhibited YAP/TAZ transcriptional activity, we evaluated the expression of the YAP/TAZ target genes, CTGF and CYR61, and found that apigenin significantly decreased the mRNA and protein levels of CTGF and CYR61 in a dose-dependent manner (Fig. 5b-d). The protein levels of YAP and TAZ, however, were not affected by apigenin treatment.

\section{Apigenin disrupted YAP/TAZ-TEAD interaction in TNBC cells}

We previously verified that the TAZ-TEADs interaction was indispensable for TAZ in maintaining CSCs traits of breast cancer cells ${ }^{25}$. Therefore, we hypothesized that apigenin could act by disrupting the interaction of TAZ and TEADs in TNBC cells, and we performed coimmunoprecipitation assays to explore the interaction between TAZ and TEADs. Apigenin disrupted the interaction of TAZ and TEADs in MDA-MB-231 and MDAMB-436 cells (Fig. 6a, b), as well as the interaction of YAP and TEADs in MDA-MB-436 cells. To further explore whether TAZ played a major role in the anticancer activities of apigenin, we decreased TAZ expression in 


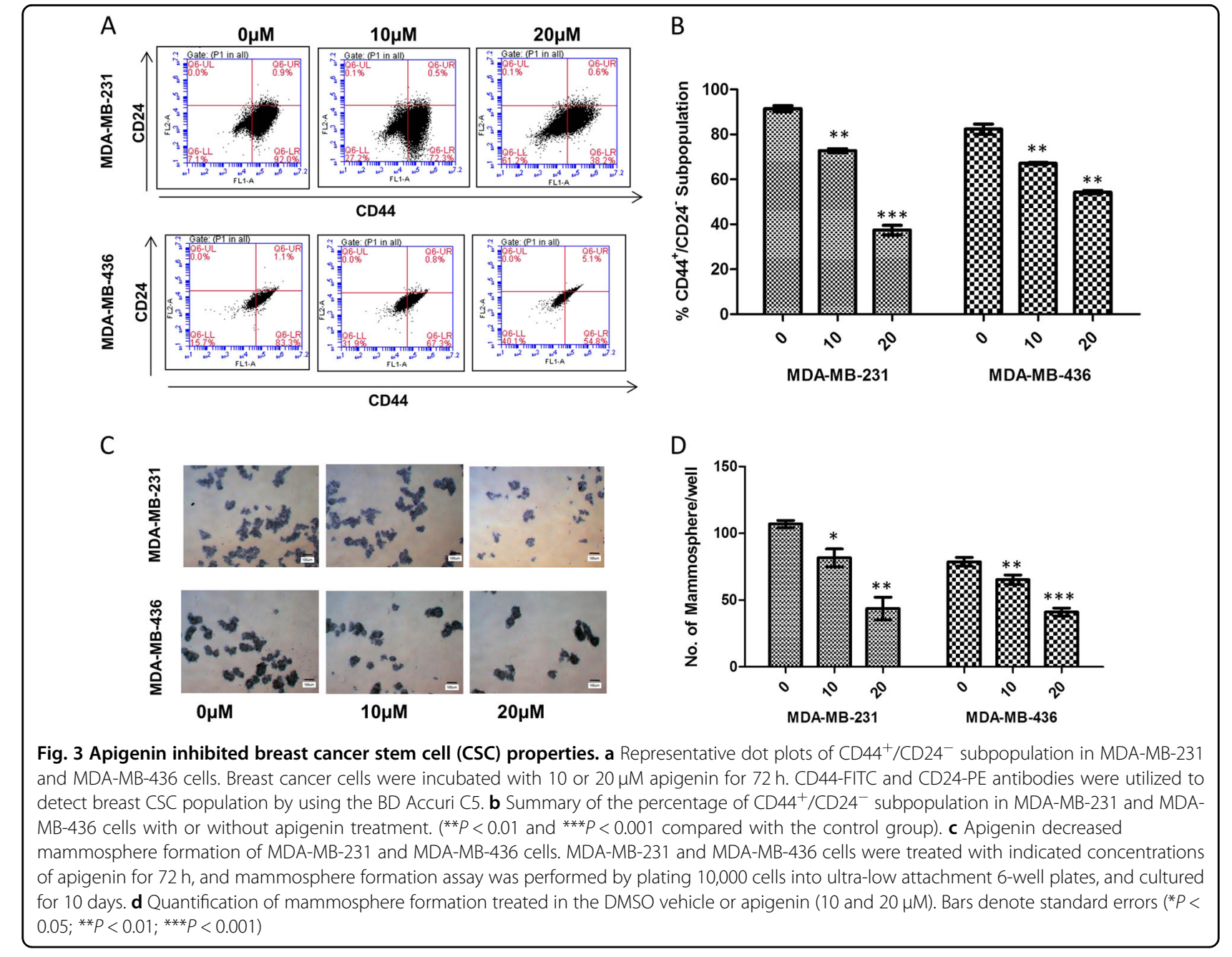

B

TNBC cells by CRISPR/Cas9-mediated genome editing. As expected, knockdown of TAZ sensitized TNBC cells to apigenin treatment, with a $50-70 \%$ decrease in IC50 (Fig. 6c, d). These results suggested that the reversion of the malignant phenotype of TNBC cells by apigenin was, at least in part, due to inhibition of the YAP/TAZ-TEADs complex activity.

\section{Discussion}

The theory of CSCs proposed that a small population of cancer cells had features similar to normal stem cells and that these cells played important roles in tumor initiation and maintenance ${ }^{26}$. In recent studies, CSCs have been isolated and identified in several solid tumors, including breast cancer ${ }^{27}$. In TNBC, breast CSCs were highly enriched and related to chemotherapy resistance, tumor relapse, and metastasis ${ }^{28-30}$. Therefore, the development of therapy targeting CSCs might benefit patients with TNBC.

Apigenin is a widely distributed flavonoid in vegetables and fruits. Recent studies have reported that apigenin has anticancer activity ${ }^{31-33}$, but the effects and underlying mechanisms of apigenin on TNBC have remained largely unexplored. In our drug-screening study, we found that apigenin decreased YAP/TAZ luciferase reporter activity. Therefore, we hypothesized that apigenin inhibited the migration and properties of CSCs in TNBC cells by regulating the Hippo-YAP/TAZ signaling pathway.

The population of $\mathrm{CD} 44^{+} / \mathrm{CD} 24^{-}$cells was recognized as CSCs in breast cancer ${ }^{34}$. In the current study, we used MDA-MB-231 and MDA-MB-436 cells to investigate the functions and mechanisms of apigenin on TNBC cells, because both cell lines overexpressed TAZ and possessed a high number of $\mathrm{CD} 44^{+} / \mathrm{CD} 24^{-}$cells. Our results showed that apigenin significantly suppressed proliferation and migration of TNBC cells. We further demonstrated that apigenin robustly inhibited features of stemness in TNBC cells, evidenced by a decrease in the $\mathrm{CD} 44^{+} / \mathrm{CD} 24^{-} \mathrm{CSC}$ subpopulation and mammosphere formation. Also, limiting dilution analysis of tumorigenesis confirmed that apigenin inhibited the tumor-initiating properties of TNBC in vivo.

Next, we investigated the molecular mechanisms underlying the anticancer effects of apigenin. We verified 


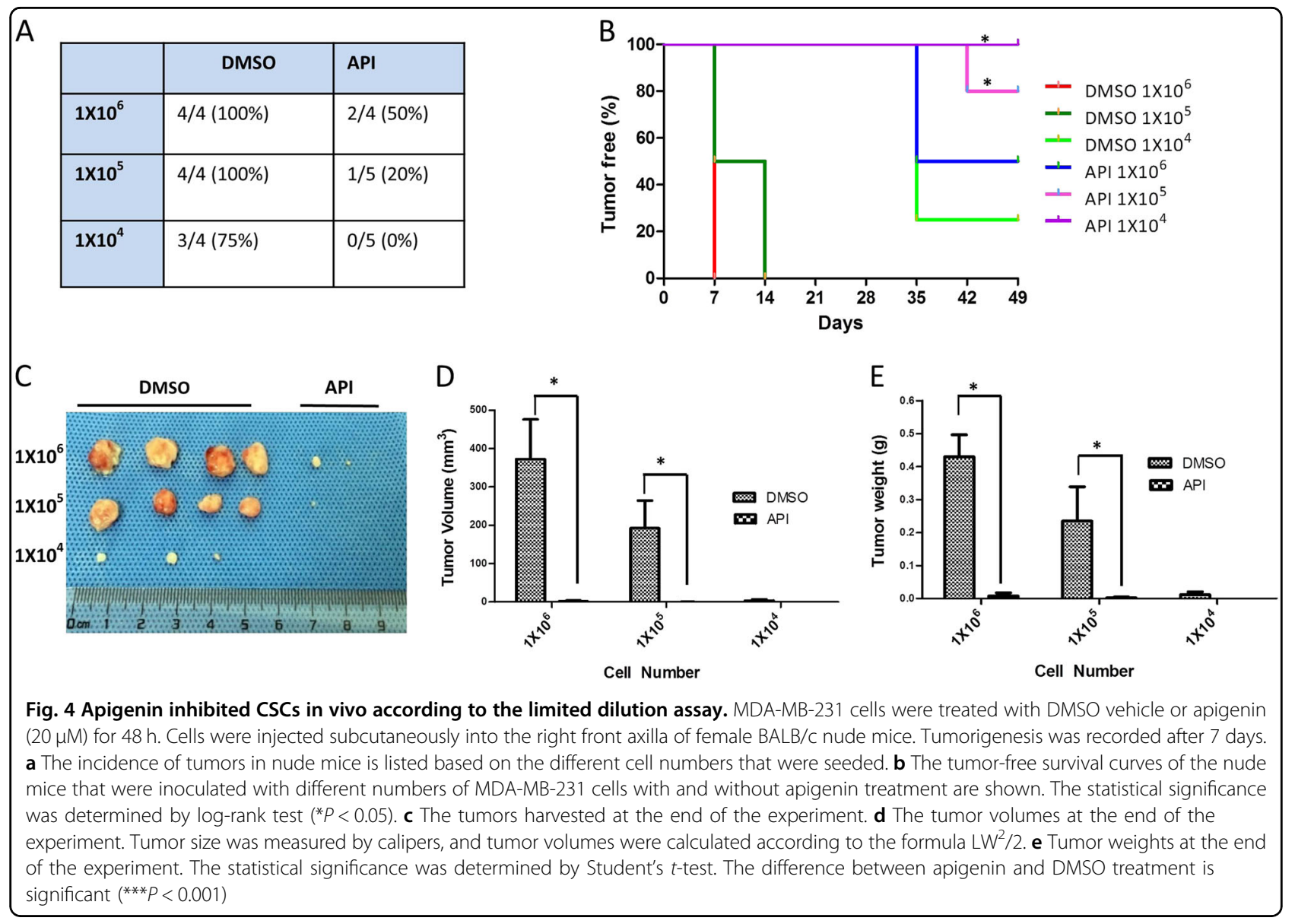

that apigenin inhibited YAP/TAZ transcriptional activity in TNBC cells, as assayed by luciferase reporter assays. Consistently, apigenin significantly decreased the expression of CTGF and CYR61, two YAP/TAZ-regulated genes, at both the mRNA and protein level.

YAP and TAZ mainly interact with TEADs to induce the expression of downstream genes that are involved in cell proliferation and migration ${ }^{35-37}$. Therefore, the YAP/ TAZ-TEADs complex may serve as a potential therapeutic target. In the current study, we investigated the effect of apigenin on the interaction of YAP/TAZ and TEADs. Our results demonstrated that apigenin disrupted the YAP/TAZ-TEADs protein-protein interaction in MDA-MB-436 cells. In MDA-MB-231 cells, we found that apigenin disrupted the TAZ-TEADs interaction. We did not find evidence for interaction between YAP and TEADs in MDA-MB-231 cells. We assume that TAZ, but not YAP, plays a key role in the downstream effects of the Hippo pathway in MDA-MB-231 cells. Furthermore, CRISPR/Cas9-mediated knockout of TAZ sensitized TNBC cells to apigenin treatment.

In conclusion, we demonstrated for the first time that apigenin inhibited YAP/TAZ activity in TNBC cells. The effects of apigenin on TNBC cells are mediated, at least in part, by disrupting the YAP/TAZ-TEADs protein-protein interaction, which is depicted in the model shown in Fig. 6e. These results suggested that apigenin might offer a novel therapeutic option for TNBC patients with high YAP/TAZ activity.

\section{Materials and methods \\ Antibodies and reagents}

YAP, TAZ, TEADs, GAPDH, $\beta$-Actin, CTGF, CYR61, rabbit $\mathrm{mAb}$ IgG control and anti-rabbit IgG (Light-Chain Specific) were purchased from Cell Signaling (Danvers, MA, United States); anti-mouse IgG and anti-rabbit IgG were purchased from Bio-Rad (Bio-Rad, Hercules, CA, United States); CD24-PE and CD44-FITC antibodies from BD Biosciences (Franklin Lakes, NJ, USA); EGF and bFGF was purchased from PeproTech, Inc. (Rocky Hill, NJ, USA), B27 and puromycin was from Thermo Fisher Scientific, Inc. (Waltham, MA, USA). BMStbl3 competent cells were from Biomed (Beijing, China); insulin, cholera toxin, hydrocortisone, ampicillin sodium salt, sulforhodamine B (SRB), LB broth, and LB broth with agar were purchased from MilliporeSigma (Burlington, MA, USA). 8xGTIIC-luciferase was a gift from Stefano Piccolo (Addgene plasmid \# 34615). Apigenin (S2262, purity 


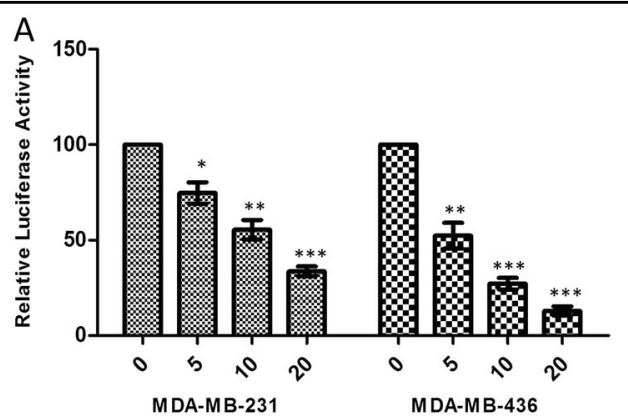

B

C

MDA-MB-436

D

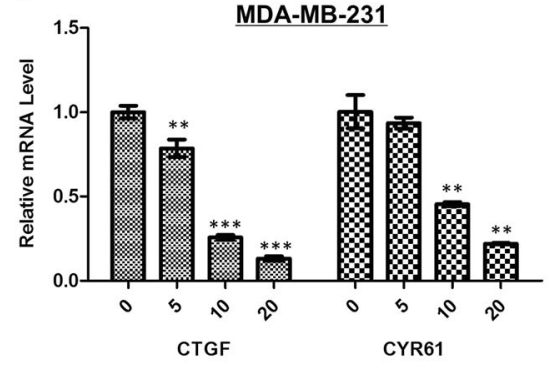

C
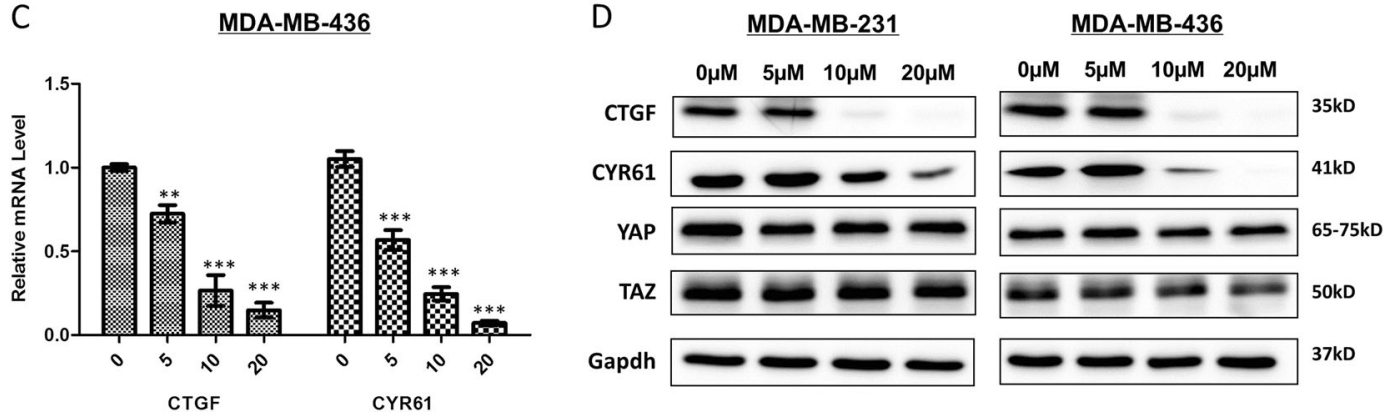

Fig. 5 Apigenin decreased YAP/TAZ transcriptional activity in TNBC cells. a Apigenin reduced YAP/TAZ luciferase reporter activity in MDA-MB231 and MDA-MB-436 cells. b, c Apigenin reduced mRNA level of CTGF and CYR61 in the two cell lines. Each experiment was performed in triplicate. The results shown are representative of at least three independent experiments. Data are shown as the means \pm S.D. $\left(* * P<0.01\right.$ and ${ }^{* *} P<0.001$ compared with the control group). $\mathbf{d}$ CTGF and CYR61 expression in apigenin-treated TNBC cells were revealed by immunoblot. Gapdh was used as loading control. The results shown are representative of at least three independent experiments

$>99 \%)$ was obtained from Selleckchem (Houston, USA) and dissolved in sterile-filtered dimethyl sulfoxide (DMSO; MP Biomedicals, Santa Ana, CA, USA). Final DMSO concentration was $0.1 \%$ in Apigenin- and vehicletreated cells. All consumables and regular reagents for the experiments were purchased from VWR Life Science (Radnor, PA, USA).

\section{Plasmid construction}

We constructed the single guide RNAs (sgRNAs) targeting exon 1 of the human WWTR1 gene (TAZ, NM_001168278) as previously described ${ }^{38}$. The target sequences for the TAZ guide were 5'-CGCGAGTGCGA GCCCGAATC-3' and 5'-GCAAGTGATCCACGTCA CGC-3'; the scrambled sequence was 5'-AACAGTCG CGTTTGCGACTGG- $3^{\prime}$. The constructs were verified by sequencing. The extraction of plasmids was performed using EndoFree Mini Plasmid Kit according to the manufacturers' protocol (TIANGEN, Beijing, China).

\section{Cell culture and transfection}

MDA-MB-231 cell lines were purchased from American Type Culture Collection (Manassas, VA, USA). MCF-10A and MDA-MB-436 cell lines were obtained from Cell Bank of Chinese Academy of Sciences. MDA-MB-231 and MDA-MB-436 cells were cultured in Dulbecco's modified Eagle medium (DMEM, GIBCO) containing 10\% fetal bovine serum (FBS). MCF-10A cells were cultured as previously described ${ }^{39}$. Cells were maintained in a humidified atmosphere of $95 \%$ air and $5 \% \mathrm{CO} 2$ at $37^{\circ} \mathrm{C}$. For knockout experiments, MDA-MB-231 and MDA-MB-436 were transfected with $2.5 \mu \mathrm{g}$ of LentiCRISPR-v2-TAZ or $2.5 \mu \mathrm{g}$ of LentiCRISPRv2-Scramble (control). Transfection was performed using Lipofectamine 3000 DNA Transfection Reagent (Thermo\#L3000-015) following the manufacturer's protocol. TAZ-edited cells and control cells were selected using $3 \mu \mathrm{g} / \mathrm{ml}$ puromycin (Gibco\#A11138-03) for $48 \mathrm{~h}$. The knockout of TAZ was validated by western blot.

\section{Cell proliferation and colony formation assay}

SRB protein assay was used in this study to analyze cell proliferation ${ }^{40}$. Briefly, TNBC cells were plated in 96well plates $\left(6 \times 10^{3}\right.$ cells per well $)$ and grown overnight. Apigenin $(0-64 \mu \mathrm{M})$ was added to the indicated wells and plates were incubated for 24,48 , or $72 \mathrm{~h}$. The IC50 value of apigenin in TNBC cells was calculated using GraphPad Software. For colony formation, MDA-MB231 and MDA-MB-436 cells (1000 cells per well) were seeded on 24-well plates. The experimental groups were treated with the indicated concentrations of apigenin for $72 \mathrm{~h}$ and then replaced for fresh medium. The plates were finally stained with $0.2 \%$ crystal violet (Amresco\#0528) and counted. 


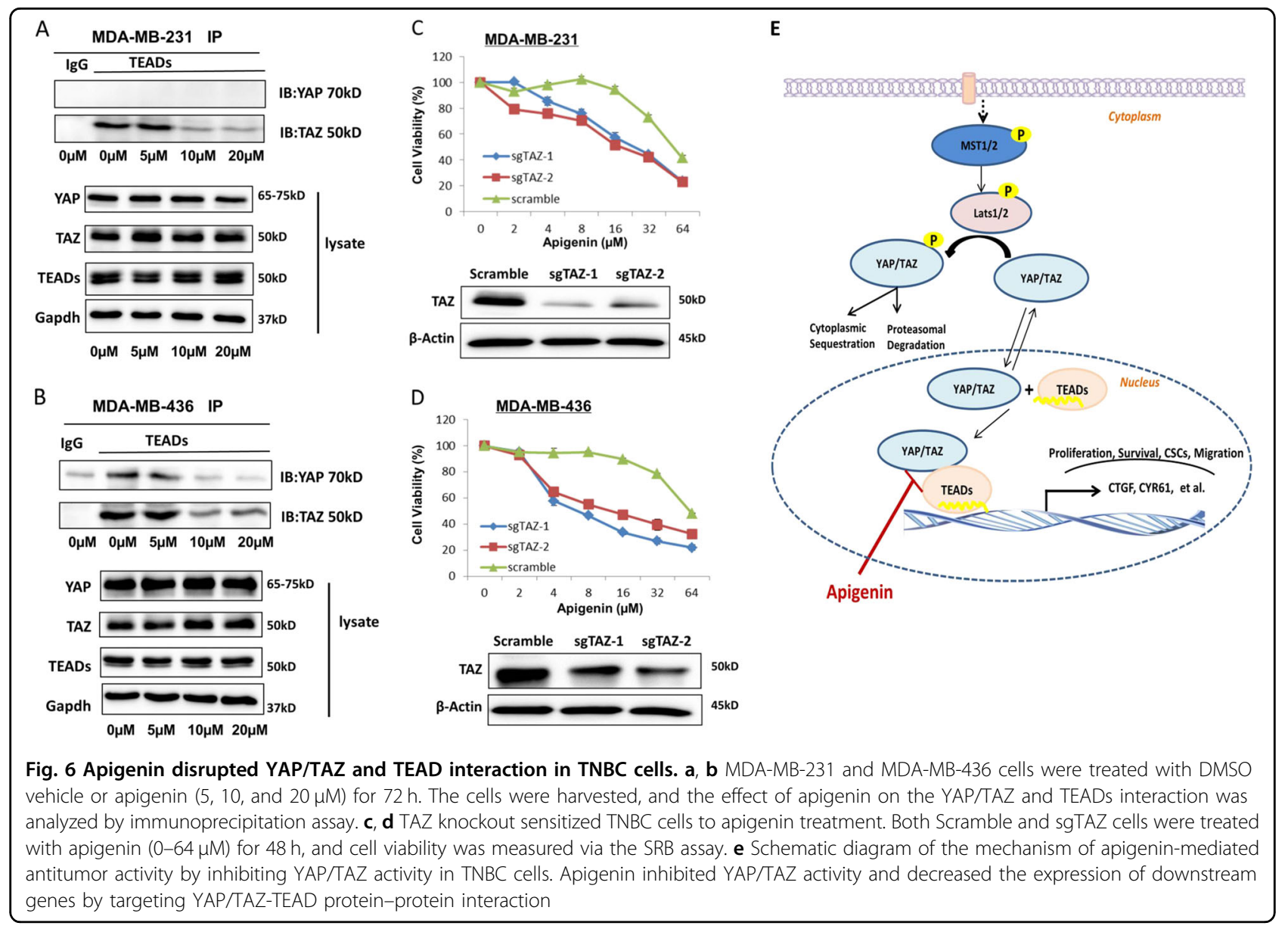

\section{Mammosphere formation assay}

TNBC cells were treated with different concentrations of apigenin for $72 \mathrm{~h}$ and mammosphere formation assay was conducted as previously decribed ${ }^{25}$. In brief, apigenin-treated and DMSO-treated cells were plated into ultra-low attachment plates at the density of 10,000 cells per well (Corning, NY, USA). On day 10, images were taken at $4 \mathrm{x}$ magnification and counted.

\section{Flow cytometry analysis}

MDA-MB-231 and MDA-MB-436 cells were treated with apigenin for $72 \mathrm{~h}$. Cells were resuspended in phosphatebuffered saline (PBS) containing 0.5\% FBS and stained with anti-CD24-PE (BD\#555428) and anti-CD44-FITC (BD\#555478) for $30 \mathrm{~min}$ at $4{ }^{\circ} \mathrm{C}$ in the dark. Cells were washed with PBS and then analyzed by flow cytometry.

\section{Wound-healing and transwell migration assays}

TNBC cells were seeded onto 6-well plates to reach 90\% confluence. A sterile P200 pipette tip was used to create a scratch in the middle of each well. Cell debris was removed, and the remaining cells were maintained in the absence or presence of apigenin for $24 \mathrm{~h}$. Migrated cells were photographed at 0,12 , or $24 \mathrm{~h}$ by using a Zeiss inverted microscope (Zeiss GmbH, Germany; magnification, $4 \times$ ). For transwell migration assay, TNBC cells were pretreated with the indicated concentrations of apigenin for $24 \mathrm{~h}$. Cells were resuspended in serum-free medium, plated into transwell chambers ( $8-\mu \mathrm{m}$ pore size; Corning), and cultured for $24 \mathrm{~h}$. Migrated cells were stained with crystal violet and counted under the microscope.

\section{Luciferase reporter assay}

Luciferase reporter assay was performed using previously described methods ${ }^{41}$. Briefly, MDA-MB-231 or MDA-MB436 cells were plated in 96-well plates and grown overnight. Cells were co-transfected with $0.2 \mu \mathrm{g}$ of 8xGTIIC-luciferase reporter plasmid and $10 \mathrm{ng}$ of pRL-CMV plasmid using Lipofectamine 3000 reagent. Indicated concentrations of apigenin $(5,10$, and $20 \mu \mathrm{M})$ were added to the cells at $24 \mathrm{~h}$ post-transfection. Luciferase activity was detected with the DLR $^{\mathrm{im}}$ assay system (Promega\#E1910), according to the manufacturers' protocol.

\section{Real-time PCR}

TRIzol reagent (Thermo, USA) was applied for total RNA extraction. The RNA was reverse transcribed using the cDNA Synthesis Kit (Thermo\#K1622) following the 
manufacturer's instructions. We performed real-time polymerase chain reaction (PCR) analysis using the Power SYBR Green PCR Master Mix Power (Roche, Mannheim, Germany) on a Bio-Rad CFX96 detection system. The mRNA levels of target genes were standardized to the level of $\beta$-Actin. Primer sequences (in the $5^{\prime}-3^{\prime}$ direction) are as follows: CTGF-F: GCAGAG CCGCCTGTGCATGG; CTGF-R: GGTATGTCTTCA TGCTGG; CYR61-F: CACACCAAGGGGCTGGAATG; CYR61-R: CCCGTTTTGGTAGATTCTGG; $\beta$-Actin-F: GGTGAAGGTCGGAGTCAACGG; $\beta$-Actin-R: GAGG TCAATGAAGGGGTCATTG.

\section{Western blotting and co-immunoprecipitation analysis}

Cell lysates were collected with RIPA buffer containing protease inhibitors (Thermo Fisher Scientific) and cleared by centrifugation. BCA Assay (Thermo\#23227) was applied for the determination of the protein concentration. Western blotting was performed according to the standard protocol. Protein bands were finally detected using the ECL detection reagents (Thermo Scientific\#34580). For co-immunoprecipitation analysis, $500 \mu \mathrm{g}$ proteins were incubated overnight with specific primary antibodies TEADs (CST\#13295) or rabbit mAb IgG control (CST\#3900) at $4{ }^{\circ} \mathrm{C}$. Immune complexes were precipitated with Protein A/G PLUS-Agarose (Santa Cruz \#sc-2003) and boiled with Laemmli sample buffer. Elutes were analyzed by Western blotting assay. The following primary antibodies were used in this study: YAP (CST\#4912), TAZ (CST\#70148), TEADs (CST\#13295), GAPDH (CST\#2118), $\beta$-Actin (CST\#3700), CTGF (CST\#86641), CYR61 (CST\#14479), and YAP/TAZ (CST\#8418).

\section{Tumorigenic evaluation assays}

MDA-MB-231 cells were pretreated with DMSO or $20 \mu \mathrm{M}$ apigenin for $48 \mathrm{~h}$. Living cells were quantified by using trypan blue exclusion assay. Cells were suspended in PBS containing 50\% Matrigel (BD\#354230). A series dilution of apigenin-treated and DMSO-treated cells $\left(10^{6}\right.$, $10^{5}, 10^{4}$ ) was injected into the right flank of female BALB/ c nude mice (5-6-weeks-old; Charles River, Beijing, China). Tumor formation efficiency and tumor sizes were recorded once a week. Tumor volumes were calculated according to the formula of Length $\times \mathrm{Width}^{2} / 2$. The care and use of animals were approved by the Animal Care and Use Committee of Guangzhou University of Chinese Medicine (Guangzhou, China).

\section{Statistical analysis of data}

GraphPad Prism 5 was used for statistical analyses. Student's $t$-test was used for comparison between groups. Data are expressed as mean \pm SD. Statistical significance was set at $P<0.05$.

\section{Acknowledgements}

This research was supported by National Natural Science Foundation of China (Grant number: 81803785) and Guangzhou University of Chinese Medicine (Grant number: A1-AFD018161Z0131).

\section{Author details}

${ }^{1}$ Tropical Medicine Institute, Guangzhou University of Chinese Medicine, 510006 Guangzhou, P.R. China. ${ }^{2}$ State Key Laboratory of Quality Research in Chinese Medicine, Macau Institute for Applied Research in Medicine and Health, Macau University of Science and Technology, Macau, P.R. China. ${ }^{3}$ The Research Center for Integrative Medicine, Guangzhou University of Chinese Medicine, 510006 Guangzhou, P.R. China. ${ }^{4}$ School of Basic Medicine Science, Guangzhou University of Chinese Medicine, 510006 Guangzhou, P.R. China. ${ }^{5}$ Guangdong Provincial Academy of Chinese Medical Sciences, Guangzhou University of Chinese Medicine, 510006 Guangzhou, P.R. China

\section{Conflict of interest}

The authors declare that they have no conflict of interest.

\section{Publisher's note}

Springer Nature remains neutral with regard to jurisdictional claims in published maps and institutional affiliations.

The online version of this article (https://doi.org/10.1038/s41420-018-0124-8) contains supplementary material, which is available to authorized users.

Received: 4 October 2018 Revised: 1 November 2018 Accepted: 5 November 2018

Published online: 20 November 2018

\section{References}

1. Fanale, D. et al. Breast cancer genome-wide association studies: there is strength in numbers. Oncogene 31, 2121-2128 (2012).

2. Siegel, R. L., Miller, K. D. \& Jemal, A. Cancer statistics, 2017. CA Cancer J. Clin. 67, 7-30 (2017).

3. Dai, X., Xiang, L., Li, T. \& Bai, Z. Cancer hallmarks, biomarkers and breast cancer molecular subtypes. J. Cancer 7, 1281-1294 (2016).

4. Foulkes, W. D., Smith, I. E. \& Reis-Filho, J. S. Triple-negative breast cancer. N. Engl. J. Med. 363, 1938-1948 (2010).

5. Anders, C. K. \& Carey, L. A. Biology, metastatic patterns, and treatment of patients with triple-negative breast cancer. Clin. Breast Cancer 9, S73-S81 (2009).

6. Cinkaya, A., Akin, M. \& Sengul, A. Evaluation of treatment outcomes of triplenegative breast cancer. J. Cancer Res. Ther. 12, 150-154 (2016).

7. Pan, D. The hippo signaling pathway in development and cancer. Dev. Cell 19, 491-505 (2010).

8. Yu, F. X. \& Guan, K. L. The Hippo pathway: regulators and regulations. Genes Dev. 27, 355-371 (2013).

9. Zanconato, F., Cordenonsi, M. \& Piccolo, S. YAP/TAZ at the Roots of Cancer. Cancer Cell 29, 783-803 (2016).

10. Zhao, B., Li, L., Lei, Q. \& Guan, K. L. The Hippo-YAP pathway in organ size control and tumorigenesis: an updated version. Genes Dev. 24, 862-874 (2010).

11. Lei, Q. Y. et al. TAZ promotes cell proliferation and epithelial-mesenchymal transition and is inhibited by the hippo pathway. Mol. Cell Biol. 28, 2426-2436 (2008).

12. Harvey, K. F., Zhang, X. \& Thomas, D. M. The Hippo pathway and human cancer. Nat. Rev. Cancer 13, 246-257 (2013).

13. Marti, P. et al. YAP promotes proliferation, chemoresistance, and angiogenesis in human cholangiocarcinoma through TEAD transcription factors. Hepatology 62, 1497-1510 (2015).

14. Lian, I. et al. The role of YAP transcription coactivator in regulating stem cell self-renewal and differentiation. Genes Dev. 24, 1106-1118 (2010).

15. Hayashi, $H$. et al. An imbalance in TAZ and YAP expression in hepatocellular carcinoma confers cancer stem cell-like behaviors contributing to disease progression. Cancer Res. 75, 4985-4997 (2015). 
16. Diaz-Martin, J. et al. Nuclear TAZ expression associates with the triple-negative phenotype in breast cancer. Endocr. Relat. Cancer 22, 443-454 (2015).

17. Kim, T. et al. A basal-like breast cancer-specific role for SRF-IL6 in YAP-induced cancer stemness. Nat. Commun. 6, 10186 (2015)

18. Liu, J. et al. Synaptopodin-2 suppresses metastasis of triple-negative breast cancer via inhibition of YAP/TAZ activity. J. Pathol. 244, 71-83 (2018).

19. Zhao, B., Tumaneng, K. \& Guan, K. L. The Hippo pathway in organ size control, tissue regeneration and stem cell self-renewal. Nat. Cell Biol. 13, 877-883 (2011).

20. Cordenonsi, M. et al. The Hippo transducer TAZ confers cancer stem cellrelated traits on breast cancer cells. Cell 147, 759-772 (2011).

21. Bartucci, $M$. et al. TAZ is required for metastatic activity and chemoresistance of breast cancer stem cells. Oncogene 34, 681-690 (2015).

22. He, X. et al. Targeting the microRNA-21/AP1 axis by 5-fluorouracil and pirarubicin in human hepatocellular carcinoma. Oncotarget 6, 2302-2314 (2015).

23. Orlikova, B. \& Diederich, M. Power from the garden: plant compounds as inhibitors of the hallmarks of cancer. Curr. Med. Chem. 19, 2061-2087 (2012).

24. Ge, J. J. et al. Two new tetracyclic triterpenoids from the barks of Melia azedarach. J. Asian Nat. Prod. Res. 18, 20-25 (2016).

25. Li, Y. W. et al. Characterization of TAZ domains important for the induction of breast cancer stem cell properties and tumorigenesis. Cell Cycle 14, 146-156 (2015).

26. Ajani, J. A., Song, S., Hochster, H. S. \& Steinberg, I. B. Cancer stem cells: the promise and the potential. Semin. Oncol. 42, S3-S17 (2015)

27. Luo, M. et al. Breast cancer stem cells: current advances and clinical implications. Methods Mol. Biol. 1293, 1-49 (2015).

28. McCarthy, N., Mitchell, G., Bilous, M., Wilcken, N. \& Lindeman, G. J. Triplenegative breast cancer: making the most of a misnomer. Asia Pac. J. Clin. Oncol. 8, 145-155 (2012).
29. Doherty, M. R. et al. Interferon-beta represses cancer stem cell properties in triple-negative breast cancer. Proc. Natl Acad. Sci. USA 114, 13792-13797 (2017).

30. Morrison, B. J., Schmidt, C. W., Lakhani, S. R., Reynolds, B. A. \& Lopez, J. A. Breast cancer stem cells: implications for therapy of breast cancer. Breast Cancer Res. 10, 210 (2008).

31. Patel, D., Shukla, S. \& Gupta, S. Apigenin and cancer chemoprevention: progress, potential and promise (review). Int. J. Oncol. 30, 233-245 (2007).

32. Nabavi, S. M., Habtemariam, S., Daglia, M. \& Nabavi, S. F. Apigenin and breast cancers: From chemistry to medicine. Anticancer Agents Med. Chem. 15 728-735 (2015).

33. Shukla, S. \& Gupta, S. Apigenin: a promising molecule for cancer prevention. Pharm. Res. 27, 962-978 (2010).

34. Yang, F., Xu, J., Tang, L. \& Guan, X. Breast cancer stem cell: the roles and therapeutic implications. Cell Mol. Life Sci. 74, 951-966 (2017).

35. Zhao, B. et al. TEAD mediates YAP-dependent gene induction and growth control. Genes Dev. 22, 1962-1971 (2008).

36. Zhang, $\mathrm{H}$. et al. TEAD transcription factors mediate the function of TAZ in cell growth and epithelial-mesenchymal transition. J. Biol. Chem. 284 13355-13362 (2009).

37. Zhou, Z. et al. Targeting Hippo pathway by specific interruption of YAP-TEAD interaction using cyclic YAP-like peptides. Faseb J. 29, 724-732 (2015).

38. Shalem, O. et al. Genome-scale CRISPR-Cas9 knockout screening in human cells. Science 343, 84-87 (2014).

39. Zhao, L., Whiteaker, J. R., Voytovich, U. J., Ivey, R. G. \& Paulovich, A. G. Antibodycoupled magnetic beads can be reused in immuno-MRM assays to reduce cost and extend antibody supply. J. Proteome Res. 14, 4425-4431 (2015).

40. Wang, W. et al. Enhanced antitumor effect of combined triptolide and ionizing radiation. Clin. Cancer Res. 13, 4891-4899 (2007).

41. Dupont, S. et al. Role of YAP/TAZ in mechanotransduction. Nature 474 179-183 (2011). 